\title{
Optimization of furfural extraction from Theobrama cacao wastes using response surface methodology
}

\author{
John Rhen $\mathrm{Uy}^{1}$, Neil Dominic Careo ${ }^{1}$, Dominick Llarena ${ }^{1}$, and John Raymond Barajas ${ }^{1, *}$ \\ ${ }^{1}$ Department of Chemical Engineering, Bicol University,Legazpi City, Albay, 4500, Philippines
}

\begin{abstract}
Cacao farming in the Philippines is continually expanding due to an influx of government support and funding. Although a comprehensive development program was implemented, the large volume of cacao biomass waste generated annually remains underutilized. In an attempt to provide a means of reusing this waste, we test the extent to which furfural can be extracted from cacao pod wastes. A boxbehnken experimental design was used to obtain the optimal conditions in the acid-catalyzed extraction of crude furfural. Extraction time (min), hydrochloric acid concentration (M), and amount of sodium chloride (g) were found to have a significant influence on the extraction yield of crude furfural. Actual values of these independent variables were chosen on the basis of preliminary experimental results. Optimum conditions using ridge analysis were found to be: extraction time $35.0 \mathrm{~min}$, hydrochloric acid concentration $5.0 \mathrm{M}$, and amount of sodium chloride $7.0 \mathrm{~g}$. Furfural extraction was also performed at optimum conditions to assess the validity of the empirical model. In conclusion, the high furfural extraction yield obtained in our experiments presents an opportunity to harness these unregulated wastes in producing high quality products.
\end{abstract}

\section{Introduction}

With the perceived decline in the global production of beans from the Theobrama cacao (cacao) tree, developing countries have been strongly encouraged to propagate cacao trees to fill the gap in the demand and supply of cocoa beans. For example, the Philippines took this opportunity and supported local cacao plantations with incentives to help this local industry to grow. The Philippine government further expressed its support to the expanding cacao industry by allowing its agencies to fully participate in the 2022 Cacao Challenge (that is to produce $100000 \mathrm{MT}$ of dried fermented cacao beans by 2022) [1]. This notion of providing incentives for developing countries therefore led to a fifty percent increase in the global production of cacao beans [2].

While the idea of using incentives to boost cacao bean production is beneficial to the local cacao industry, this also propagates the production of undesirable waste residues in cacao farms and plantations. It has been reported that about $52-76 \%$ of the whole cacao fruit are considered as undesirable solid residues in the form of cacao pods (CP) [3-4]. In fact, a ton of processed cocoa beans approximately generates 10 tons of CP solid residues [5]. Traditionally, $\mathrm{CP}$ residues are allowed to freely decompose in a secluded but open area within cacao plantations to mitigate possible environmental complications. However, untreated decomposing CP residues elevate the risk of spreading botanical diseases such as black pod rot [5-6]. The large volume of CP residues generated from the cacao fruit then presents a

\footnotetext{
* Corresponding author: jrbarajas12@gmail.com
}

challenging waste management problem that must be immediately addressed.

Converting CP into furfuraldehyde (furfural) is an economical and promising way of reducing the volume of these waste residues into manageable levels. Furfural is a chemical with various applications in the field. For instance, furfural can be used as a selective solvent to refine petroleum, lubricating oils, diesel fuels, and vegetable oils [7]. Furfural can be also used as a starting chemical reagent to produce high value added products such as furfuryl alcohol and tetrahydrofurfuryl alcohol [8]. In the present work, we investigate the extent to which $\mathrm{CP}$ residues can be converted into furfural. To assess the suitability of $\mathrm{CP}$ residues as a precursor material for furfural production, we implemented a boxbehnken experimental design to achieve an optimum yield of furfural derived from extracts of $\mathrm{CP}$ residues.

\section{Materials and methods}

\subsection{Materials and reagents}

Cacao (Theobroma cacao L.) pods used in this study were acquired from local markets in Barangay BinodegahanPio Duran Albay, Philippines. Fresh cacao pods were selected according to criteria described in Philippine National Standards for Cacao Beans [9]. The average particle size of the cacao pods was reduced to 1 $\mathrm{cm}$. These smaller pods were then oven-dried for 5 hours at $110^{\circ} \mathrm{C}$ to remove excess moisture. After drying, the dried pods were ground to finer particles and sieved 
through a $1 \mathrm{~mm}$ screen. Analytical grade reagents $(\mathrm{HCl}$ and $\mathrm{NaCl}$ ) used for acid hydrolysis were purchased from Belman Laboratories. Furfuraldehyde standard was purchased separately from Haihang Industry Co., Ltd. Distilled water was used in all experiments.

\subsection{Determination of furfuraldehyde aqueous concentration}

Furfural solutions with concentrations ranging from 50$300 \mathrm{ppm}$ were prepared from the standard solution of furfuraldehdye. Aqueous concentrations of furfural were checked by performing a full wavelength scan using a Perkin Elmer Lambda $45 \mathrm{UV}-\mathrm{V}$ is spectrophotometer. Highest absorbance was recorded at a wavelength of 276 $\mathrm{nm}$. A calibration curve was then prepared to determine furfural concentrations from cacao pod extracts.

\subsection{Acid hydrolysis experimental set-up}

Procedure to hydrolyze cacao pods to furfuraldehydewas adopted from published literature [7, 10]. $10 \mathrm{~g}$ of dried pods and $\mathrm{NaCl}$ of known amount $(6.0,7.0$, or $8.0 \mathrm{~g})$ were mixed in the distilling flask. $100 \mathrm{~mL}$ of $\mathrm{HCl}$ solution (4.0, 4.5, or $5.0 \mathrm{M}$ ) was then carefully added into the flask (see Fig. 1). The resulting mixture was moderately agitated for 5 minutes to equally disperse the biomass in solution. The solution was heated to $160^{\circ} \mathrm{C}$ and agitation was continually induced to equally distribute heat in solution. Once the solution starts to boil, the distillate was condensed into liquid and collected in a receiving flask. The condensed distillate obtained was a clear yellow liquid. This set-up was conducted in a dark room to minimize furfural reactivity.

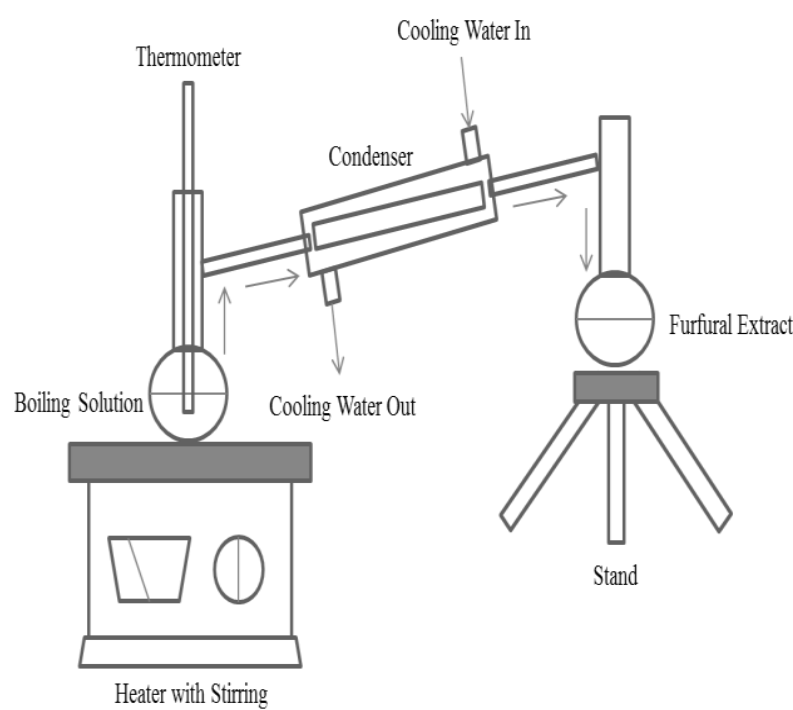

Fig. 1. Cacao Pod Acid Hydrolysis Experimental Set-up

\subsection{Experimental design}

A box-behnken experimental design (see Table 1 and Table 2) generated using Stat-Ease design expert software was implemented to investigate the influence of independent variables $(\mathrm{HCl}$ concentration $\mathrm{M}$; amount of
$\mathrm{NaCl} \mathrm{g}$; residence time min) in optimizing the desired response (furfural yield $\mathrm{mg} / \mathrm{L}$ ). All experimental runs were performed in duplicates.

Table 1. Independent Variables Influencing Furfural Yield

\begin{tabular}{llcc}
\hline \multirow{2}{*}{ Factor } & \multirow{2}{*}{ Unit } & \multicolumn{2}{c}{ Factor level } \\
\cline { 3 - 4 } & & Low & High \\
\hline HCl Cocentration & $\mathrm{M}$ & 4.0 & 5.0 \\
Amount of $\mathrm{NaCl}$ & $\mathrm{g}$ & 6 & 8 \\
Residence Time & $\mathrm{min}$ & 35 & 45 \\
\hline
\end{tabular}

Table 2. Box-Behnken Design Experimental Runs

\begin{tabular}{|c|c|c|c|}
\hline Run & $\begin{array}{c}\text { Factor } 1 \mathrm{~A}: \mathrm{HCl} \\
\text { Concentration } \\
(\mathrm{M})\end{array}$ & $\begin{array}{c}\text { Factor } 2 \mathrm{~B}: \\
\text { Amount of } \mathrm{NaCl} \\
(\mathrm{g})\end{array}$ & $\begin{array}{c}\text { Factor 3 C: } \\
\text { Residence Time } \\
\text { (min) }\end{array}$ \\
\hline 1 & 4 & 6 & 40 \\
\hline 2 & 5 & 7 & 45 \\
\hline 3 & 5 & 7 & 35 \\
\hline 4 & 4.5 & 8 & 35 \\
\hline 5 & 4.5 & 6 & 45 \\
\hline 6 & 4.5 & 8 & 45 \\
\hline 7 & 5 & 6 & 40 \\
\hline 8 & 4 & 8 & 40 \\
\hline 9 & 4.5 & 7 & 40 \\
\hline 10 & 4.5 & 6 & 35 \\
\hline 11 & 4 & 7 & 35 \\
\hline 12 & 4.5 & 7 & 40 \\
\hline 13 & 5 & 8 & 40 \\
\hline 14 & 4 & 7 & 45 \\
\hline 15 & 4.5 & 7 & 40 \\
\hline 16 & 4.5 & 7 & 40 \\
\hline 17 & 4.5 & 7 & 40 \\
\hline
\end{tabular}

\section{Results and discussion}

\subsection{Preliminary experiments}

To determine the possible factor levels that would be used for optimization, preliminary experiments were conducted. Working levels for $\mathrm{HCl}$ concentration, amount of $\mathrm{NaCl}$, and residence time were investigated by implementing a one-factor-at-a-time (OFAT) methodology. The possible ranges identified where furfural extracted from $\mathrm{CP}$ residues would be maximized are summarized in Table 3. Based on the results of these experiments (data not shown), furfural yields were found to be high (between 180 and $220 \mathrm{ppm}$ ) within the following working range: $\mathrm{HCl}$ Concentration $=4-5 \mathrm{M}$; amount of $\mathrm{NaCl}=6-8 \mathrm{~g}$; and residence time $=35-45$ $\min$. Hence, these were used as the working factor levels for the response surface methodology applied in this study. 
Table 3. Levels Tested in Preliminary Experiments

\begin{tabular}{lcc}
\hline \multicolumn{1}{c}{ Factor } & Range & Unit \\
\hline $\mathrm{HCl}$ concentration & $1-5$ & $\mathrm{M}$ \\
Amount of $\mathrm{NaCl}$ & $2-8$ & $\mathrm{~g}$ \\
Residence Time & $15-45$ & $\mathrm{~min}$ \\
\hline
\end{tabular}

\subsection{Statistical tests and mathematical model building}

To maximize the amount of furfural that can be extracted from the $\mathrm{CP}$, a response surface methodology was implemented in this study. A box-behnken design with three-factors and 12 experimental runs was adopted. Using Design Expert 11 Software ${ }^{\circledR}$ (Stat-Ease, USA), 5 center points were also added to measure the degree of variability and stability of the response variable (furfural yield). A second-order mathematical model was then generated by the software used. The model (pvalue $<0.0001$ ) with the actual factors is given in Eq. 1 .

$$
\begin{aligned}
& \text { Furfural Yield } \\
& \qquad \begin{aligned}
& \text { Intercept }+C_{1} A+C_{2} B+C_{3} C+C_{4} A B \\
& \quad+C_{5} A C+C_{6} B C+C_{7} A^{2}+C_{8} B^{2}+C_{9} C^{2}
\end{aligned}
\end{aligned}
$$

where A corresponds to the factor $\mathrm{HCl}$ concentration (M), $\mathrm{B}$ is the amount of $\mathrm{NaCl}(\mathrm{g}), \mathrm{C}$ is the residence time (min), and $\mathrm{C}_{1}$ to $\mathrm{C}_{9}$ are coefficients with values summarized in Table 4.

Table 4. Values of Coefficients in the Empirical Model

\begin{tabular}{cccc}
\hline Term & $\begin{array}{c}\text { Coefficient } \\
\text { Value }\end{array}$ & p-value & Remarks \\
\hline Intercept & -511.92 & - & - \\
A & -653.52 & 0.002511 & Significant \\
B & 927.12 & 0.003465 & Significant \\
C & -47.23 & 0.2986 & Not Significant \\
AB & -64.80 & 0.001833 & Significant \\
AC & -6.92 & 0.07378 & Not Significant \\
BC & 0.97 & 0.6055 & Not Significant \\
A & 158.66 & 0.0002 & Significant \\
B & -49.68 & $<0.0001$ & Significant \\
C $^{2}$ & 0.91 & 0.01841 & Significant \\
\hline
\end{tabular}

Table 5 summarizes the predicted and observed values for the furfural yield. It was noted that the predicted values are in good agreement with the observed values. To further examine whether the predicted values were valid, the software was used to assess the goodness of fit of the empirical model.
Table 5. Observed and Predicted Values from the BoxBehnken Experimental Design

\begin{tabular}{ccccccc}
\hline Run & A & B & C & Observed & Predicted & \% Error \\
\hline 1 & -1 & -1 & 0 & 340.80 & 327.50 & -3.90 \\
2 & 1 & -1 & 0 & 454.11 & 436.44 & -3.89 \\
3 & -1 & 1 & 0 & 332.21 & 349.87 & 5.32 \\
4 & 1 & 1 & 0 & 315.93 & 329.23 & 4.21 \\
5 & -1 & 0 & -1 & 386.34 & 386.92 & 0.15 \\
6 & 1 & 0 & -1 & 460.71 & 465.66 & 1.07 \\
7 & -1 & 0 & 1 & 440.37 & 435.42 & -1.12 \\
8 & 1 & 0 & 1 & 445.56 & 444.97 & -0.13 \\
9 & 0 & -1 & -1 & 350.29 & 363.00 & 3.63 \\
10 & 0 & 1 & -1 & 329.15 & 310.90 & -5.55 \\
11 & 0 & -1 & 1 & 348.97 & 367.22 & 5.23 \\
12 & 0 & 1 & 1 & 347.20 & 334.48 & -3.66 \\
13 & 0 & 0 & 0 & 387.64 & 370.77 & -4.35 \\
14 & 0 & 0 & 0 & 392.23 & 370.77 & -5.47 \\
15 & 0 & 0 & 0 & 389.00 & 370.77 & -4.68 \\
16 & 0 & 0 & 0 & 343.07 & 370.77 & 8.08 \\
17 & 0 & 0 & 0 & 341.93 & 370.77 & 8.44 \\
\hline
\end{tabular}

As summarized in Table 6, $\mathrm{R}^{2}$ values were very close to unity and strongly indicate that the second-order response model has a good fit. Furthermore, the adjusted and predicted $\mathrm{R}^{2}$ are also in good agreement with each other. These results highlight that there is a strong correlation between the predicted and observed outcomes.

Table 6. Statistical Fit of the Empirical Model

\begin{tabular}{cccc}
\hline \multicolumn{4}{c}{ Fit Statistics } \\
\hline Std. Dev. & 26.17 & $\mathbf{R}^{\mathbf{2}}$ & 0.7939 \\
Mean & 376.79 & Adjusted $\mathbf{R}^{2}$ & 0.7167 \\
C.V. \% & 6.94 & Predicted R $^{2}$ & 0.6337 \\
\hline
\end{tabular}

\subsection{Optimization of pectin recovery}

The furfural extraction process employed in this study was optimized by using the validated mathematical model. The suitable conditions for achieving a maximum furfural yield are presented in Fig. 2. These represented the factor combination with the highest desirability. Essentially, based on the verified model, desirability close to unity would give a response close to the experimental value. Furthermore, as shown in Fig. 3, the 3D response surface gave an estimated furfural yield of $465.66 \mathrm{ppm}$. To test whether this predicted value would be in close agreement to the observed value, confirmatory runs were also done. It was noted that an error between $8-19 \%$ was incurred. The high variability obtained may be due high reactivity of furfural with the environment. Nevertheless, the validated model has still a good potential to predict furfural yields within the working ranges specified in this study. 

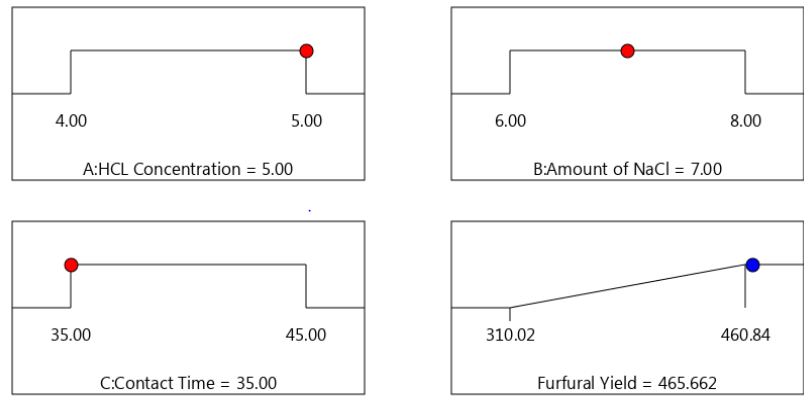

Fig. 2. Factor Level Combination to Maximize Furfural Yield

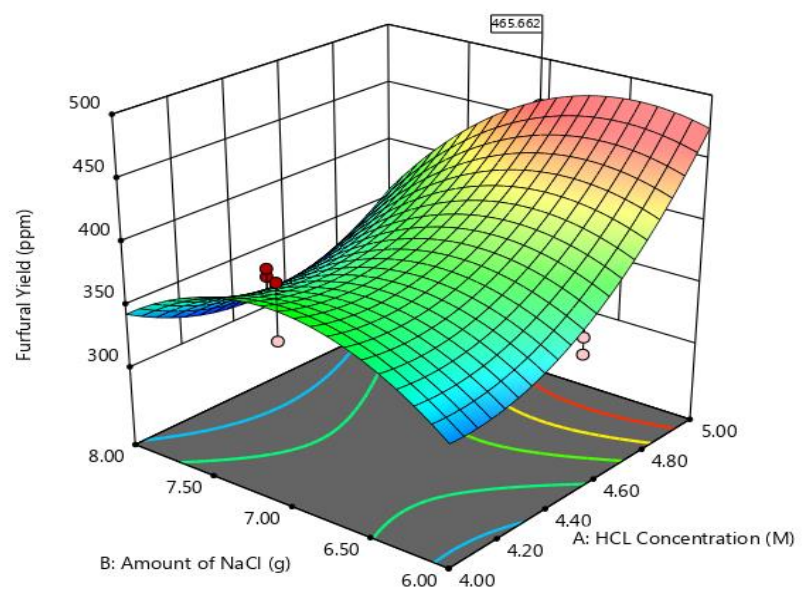

Fig. 3. 3D Response Surface for Furfural Yield

\section{Conclusion}

The use of $\mathrm{CP}$ wastes as raw materials for furfural production is an economical way of managing the increasing volume of CP annually. In the present work, we have provided evidences that $\mathrm{CP}$ can effectively be converted into furfural. We have also noted that a relatively high furfural yield (460.71 ppm) was achieved experimentally. This obtained response was replicated by using the empirical model generated. Optimum conditions were found to be: $\mathrm{HCl}$ concentration $=5.0 \mathrm{M}$; amount of $\mathrm{NaCl}=7.0 \mathrm{~g}$; residence time $=35.0 \mathrm{~min}$.

We would like to thank Bicol University College of Science for allowing us to use their UV-Vis spectrophotometerand Bicol University College of Engineering - Chemical Engineering Department for granting us permission to use their facilities.

\section{References}

[1] Available online at http://bpi.da.gov.ph/bpi/ima ges/PDF_file/Cacao\%20Industry\%20Roadmap $\% 2$ 0 -

\%20Signed\%20\%20\%20March\%2010,\%202017. pdf

[2] Wessel M. and Quist-Wessel P.M. Cocoa production in west Africa, a review and analysis of recent developments, NJAS-Wgenigen Journal of Life Sciences 74, 2015, 1-7.
[3] Chan S.Y., and Choo W.S. Effect of extraction conditions on the yield and chemical properties of pectin from cocoa husks, Food Chemistry 141(4), 2013, 3752-3758.

[4] Donkoh A., Atuahene C. C., Wilson B. N., and Adomako D. Chemical composition of cocoa pod husk and its effect on growth and food efficiency in broiler chicks, Animal Feed Science and Technology 35(1-2), 1991, 161-169.

[5] Figueira A., Janick J., and BeMiller J. N. Partial characterization of cacao pod and stem gums, Carbohydrate Polymer 24(2), 1994, 133138.

[6] Barazarte H., Sangronis E., and Unai E. Cocoa (Theobroma cacao L.) hulls: a posible commercial source of pectins, ArchivosLatinoamericanos de Nutricion58(1), 2008, 64-70.

[7] Gebre H., Fisha K., Kindeya T., and Gebremical T. Synthesis of furfural from bagasse, International Letters of Chemistry, Physics and Astronomy 57, 2015, 72-84.

[8] Dutta S., De S., Saha B., and Alam M.I. Advances in Conversion of Hemicellulosic Biomass to Furfural and Upgrading to Biofuels, Catalysis Science and Technology, 2, 2012, 2025-2036.

[9] Available online at http://bafps.da.gov.ph

[10] Wankasi D., Naidoo E.B., and Bayelsa S. Furfural Production from the Epicrap of Wild Mango ( Irvingia Species ) Fruits by Acid Catalyzed Hydrolysis, ScienceHuß, 2012,pp. 47-50. 